\title{
Effects of strain induced crystallization on the segmental dynamics of vulcanized natural rubber
}

\author{
Marianella Hernández ${ }^{*}$, Miguel A. López-Manchado', Alejandro Sanz ${ }^{2}$, Aurora \\ Nogales $^{2}$, Tiberio A. Ezquerra ${ }^{2 *}$ \\ ${ }^{1}$ Instituto de Ciencia y Tecnología de Polímeros, ICTP-CSIC, Juan de la Cierva 3, Madrid 28006, Spain. \\ ${ }^{2}$ Instituto de Estructura de la Materia, IEM-CSIC, Serrano 121, Madrid 28006, Spain. \\ *corresponding authors: marherna@ictp.csic.es or imte155@iem.cfmac.csic.es
}

\begin{abstract}
The effects of strain-induced crystallization on the segmental dynamics of vulcanized natural rubber (NR) have been studied by combining dielectric relaxation spectroscopy and wide-angle X-ray scattering. The segmental dynamics of vulcanized NR is clearly affected by uniaxial stretching. Upon stretching three regimes are detected. For low strains, $\lambda<3$, stretching takes place without crystallization. However, a dramatic increase of the dielectric strength, $\Delta \varepsilon$, is observed which has been explained by an increase of the effective dipole moment upon stretching. This effect is accompanied by an increment of the fragility parameter, $m$, since as free volume reduces by stretching more cooperativity is needed in order to accomplish segmental motions. In the second regime, $3<\lambda<7.5$, crystals develop and the inclusion of segments into the crystalline phase counteracts the increment in $\Delta \varepsilon$ provoked by stretching. Our results support previous morphological model indicating that during strain induced crystallization shorter chains are progressively incorporated into the crystals while a significant amount of longer chains remains rather coiled.
\end{abstract}

Keywords: natural rubber, segmental dynamics, strain-induced crystallization, dielectric spectroscopy.

\section{Introduction}

Natural rubber (NR) has been recognized as a fascinating industrial material that has both, high elasticity and tensile strength. It is one of the most important natural materials, which has been widely used in industry as well as in our daily life, e.g., pneumatic tires, tubes, films like surgical gloves, rubber bands, etc. This versatility is derived from its toughness based on the outstanding tensile properties and excellent crack growth resistance. ${ }^{1-4}$

Moreover, the good performance of vulcanized NR products has been thought to originate from the ability to crystallize when subjected to an extensional field. The enhancement of tensile modulus along with the superior stress at break and tear strength 
under fast deformation should be related to the formation of strain-induced crystals. These crystals, embedded into an amorphous phase, are tens of nanometers in size and may orient themselves in response to macroscopic loading. From the point of view of composite materials, the crystals act like fillers which form in situ upon deformation. ${ }^{1,5-}$ ${ }^{7}$ For this reason strain-induced crystallization of NR still attracts a lot of interest. ${ }^{2,8-12}$

In many elastomeric applications, network chain dynamics under external stress/strain are critical for determining ultimate performance. Thus, a study on how the straininduced crystallization affects the dynamics of a rubbery material is of outmost importance. Lee et al. ${ }^{13}$ reported their initial findings on the role of uniaxial extension on the relaxation behaviour of cross-linked polyisoprene (PI) by means of dielectric spectroscopy. They found that the segmental process of lightly crosslinked PI is not affected by uniaxial deformation up to an extension ratio below $\lambda=4$. Nonetheless, to our best knowledge no in-depth study of the effects of strain induced crystallization on the molecular dynamics of vulcanized NR has been undertaken. In particular, it remains unknown the interrelationship between molecular dynamics and deformation in vulcanized NR. Broadband dielectric spectroscopy (BDS) has been proven to be a powerful technique to investigate both segmental and chain dynamics of natural and synthetic rubbers. ${ }^{14-16}$ In addition, the combination of BDS with X-ray scattering techniques has revealed as an efficient approach to deal with order-dynamics relationships. ${ }^{17,18}$ The main objective of the present work is to understand the effect of strain induced orientation on the segmental dynamics of vulcanized NR. We have focused our study in the effect of network structure and the strain-induced crystallization, as investigated by synchrotron X-ray scattering, in the segmental dynamics of NR assessed by BDS.

\section{Experimental Section}

\subsection{Materials}

Natural rubber (NR) (composed of cis-1,4-isoprene units ${ }^{19}$ ) was kindly supplied by Malaysian Rubber (Berhad, Malaysia) under the trade name SMR CV60 (Mooney viscosity: $\left.\mathrm{ML}(1+4) 100{ }^{\circ} \mathrm{C}=60\right)$. The compounding ingredients were all commercial grades and were added to the rubber. The formulation and ingredients used to prepare vulcanized NR are compiled in Table 1.

Table 1. Formulation and ingredients used in the preparation of vulcanized NR.

\begin{tabular}{lc}
\hline Ingredient & Parts per hundred of rubber (phr) \\
\hline NR & 100.0 \\
Zinc oxide & 5.0 \\
Stearic acid & 1.0 \\
MBTS $^{(1)}$ & 1.0 \\
Sulfur & 2.5 \\
${ }^{(1)}$ mercapto benzothiazyl disulfide &
\end{tabular}




\subsection{Sample Preparation}

All ingredients were mixed in an open two-roll laboratory mill at room temperature. The rotors operated at a speed ratio of 1:1.4. Rubber was vulcanized in an electrically heated hydraulic press. NR films, $0.25 \mathrm{~mm}$ thick, were cured at $150{ }^{\circ} \mathrm{C}$ for $15 \mathrm{~min}$ according to the optimum cure time ( $\left.t_{90}\right)$ derived from the curing curves previously determined by means of a Rubber Process Analyzer (RPA2000 Alpha Technologies). Rectangular-shaped specimens were mechanically cut out from the vulcanized film samples.

\subsection{Experimental Methods}

The uniaxial deformation of vulcanized NR specimens was performed in a dynamometer (Instron, model 3366) at $25^{\circ} \mathrm{C}$, and at a cross-head speed of $500 \mathrm{~mm} / \mathrm{min}$. Samples with strain ratios $(\lambda)$ from 0 to 7.5 were prepared. All specimens were stretched by the movable clamp until the desired strain was achieved. Subsequently, each sample was held at the selected elongation, and glued either to a gold-plated electrode for BDS measurements, as shown in Figure 1, or to a metallic frame for the Xray scattering experiments. The strained sample-electrode (frame) assembly was then separated from the dynamometer clamps.

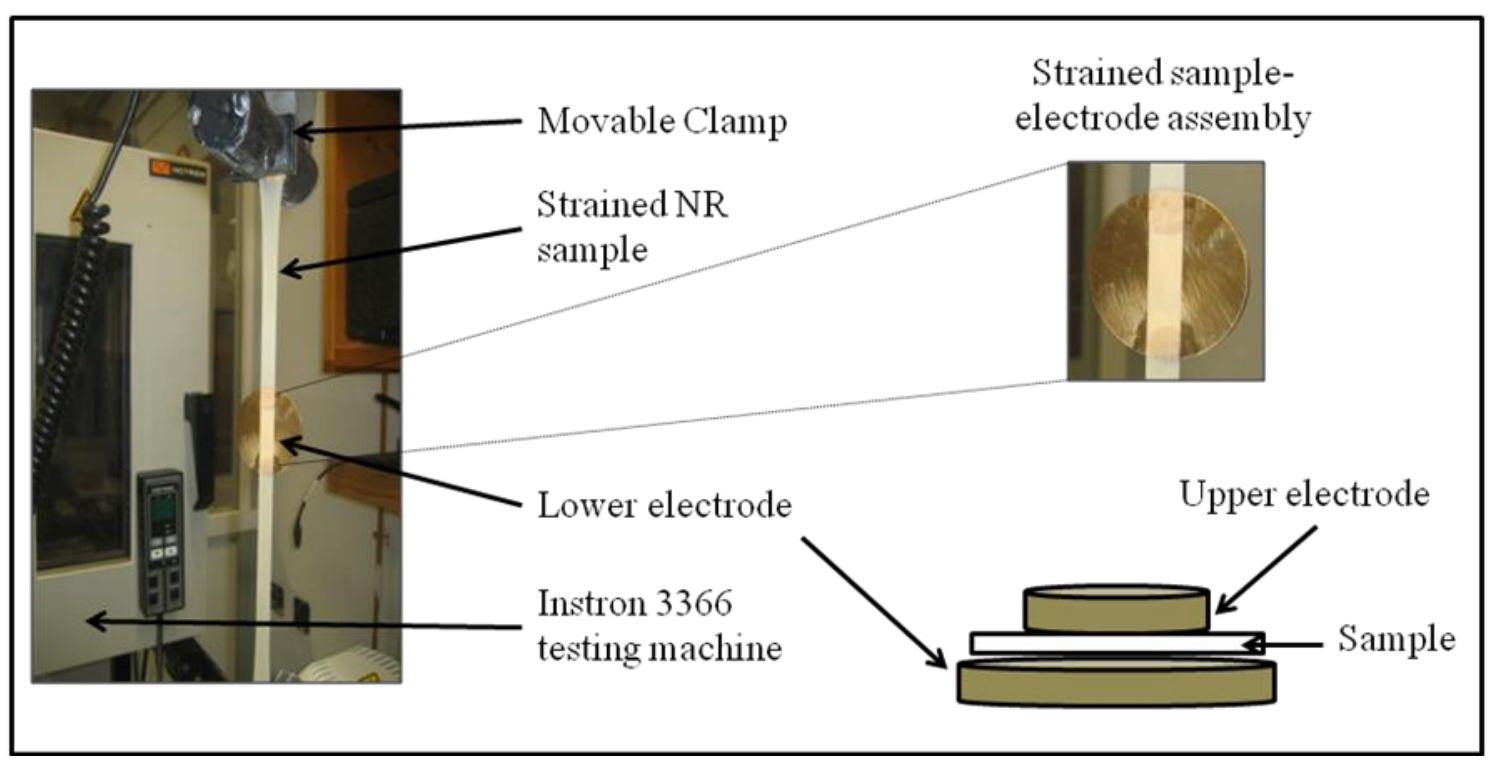

Figure 1. Schematic picture of strained sample-electrode assembly corresponding to a strain ratio $\square \lambda=7.5$. 
Wide angle X-ray scattering (WAXS) measurements of the stretched samples were carried out at the BM16 beam line ${ }^{20}$ in the European Synchrotron Radiation Facility (ESRF) in Grenoble, France. Each stretched sample-frame assembly was placed in the beam path. The wavelength of the X-rays used was $0.9795 \AA$. The two-dimensional WAXS patterns were recorded using a MarCCD camera (X-Ray Research, GmbH, Norderstedt, Germany). The image acquisition time for each frame was $3 \mathrm{sec}$. The diffraction angle in WAXS was calibrated by a standard sample of alumina. All measured images were corrected for background, beam fluctuations and sample absorption. The data analysis software FIT2 ${ }^{\odot}$ was used to analyze the WAXS images.

Broadband dielectric spectroscopy (BDS) measurements were performed on an ALPHA $\square \square$ high resolution dielectric analyzer (Novocontrol Technologies GmbH, Hundsangen, Germany). The stretched sample-electrode assembly was mounted in the dielectric cell between two parallel gold-plated electrodes. The complex permittivity $\varepsilon^{\star}$ of a given sample was calculated from the measurement of the complex impedance ${ }^{16}$. The complex permittivity of the stretched samples was measured over a frequency window of $10^{-1}<\mathrm{F} / \mathrm{Hz}<10^{7}(\mathrm{~F}=\omega / 2 \pi$ is the frequency of the applied electric field being $\omega$ the angular frequency) in the temperature range from -100 to $100{ }^{\circ} \mathrm{C}$ in $5{ }^{\circ} \mathrm{C}$ steps. The imaginary part $\varepsilon^{\prime \prime}$ of the obtained dielectric permittivity, referred to as dielectric loss, was analyzed by the phenomenological Havriliak-Negami (HN) function $^{21,22}$

$$
\varepsilon^{*}(\omega)=\varepsilon_{\infty}+\frac{\varepsilon_{s}-\varepsilon_{\infty}}{\left.1+\left(i \omega \tau_{\mathrm{HN}}\right)^{b}\right]^{c}}
$$

Where $\Delta \varepsilon=\varepsilon_{s}-\varepsilon_{\infty}, \varepsilon_{\infty}$ and $\varepsilon_{s}$ are the unrelaxed and relaxed values of the dielectric constant, $\tau_{\mathrm{HN}}$ is a characteristic relaxation time, and $b$ and $c$ are shape parameters $(0<b$, $c \leq 1$ ) which describe the symmetric and the asymmetric broadening of the equivalent relaxation time distribution function, respectively.

\section{Results}

\subsection{Strain induced crystallization}

The tensile stress-strain curve of the vulcanized NR investigated here is shown in Figure 2. As expected, the mechanical behaviour corresponds to an elastomer exhibiting rubber-like elasticity. In this case, the application of stress is considered to cause molecules to change from a coiled to an extended configuration instantaneously. The behaviour is Hookean, with a linear relationship between stress and strain only at low strains. ${ }^{23}$ A noticeable upturn in stress is observed as stretching proceeds, specifically after $\lambda=3$, characterized by a change in the slope of the stress-strain curve. This characteristic property of NR is believed to be due to a crystallization process induced 
by stretching causing molecular alignment in the stretching direction. This peculiar effect, referred to as strain induced crystallization, ${ }^{24}$ provides vulcanized NR a selfreinforcement character which has been recognized as the main factor responsible for the toughness of the material. It is thought that strain-induced crystallites form an additional physical cross-linking network, bearing most of the applied load. In order to quantitatively evaluate the strain induced crystallization in the investigated vulcanized NR under uniaxial stretching, wide-angle X-ray (WAXS) diffraction patterns were measured for samples stretched at different strain ratios (from 0 to 7.5), as shown in Figure 2. For the initial undeformed sample (inset (a)), the pattern exhibits a halo characteristic of an amorphous isotropic material. A similar situation is observed even at $\lambda=2.5$ (inset (b)). However, at $\lambda=3$ (inset (c)) the presence of crystalline diffraction maxima can be already detected. Thus, we can assume that below $\lambda=3$ mainly orientation of polymer chains occurs. For strains higher than $\lambda=3$ (insets (d-g)), the WAXS patterns clearly show the diffraction maxima characteristic of an oriented crystalline phase revealing the occurrence of strain induced crystallization. Moreover, the peak intensities of these reflections increase with increasing strain. This effect can be better visualized by the $360^{\circ}$ azimuthal integration of the WAXS patterns represented in Figure 3(a). Here the $360^{\circ}$ azimuthally integrated intensity has been represented as a function of the scattering vector $\mathrm{q}=4 \pi / \lambda(\sin \theta)$ being $\lambda$ the wavelength and $2 \theta$ the scattering angle. It is noteworthy that an amorphous halo remains during the stretching process suggesting the presence of a significant amorphous phase during stretching.

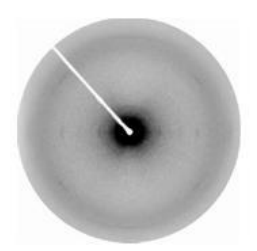

(c)

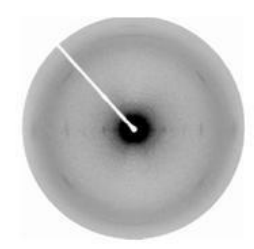

(d)

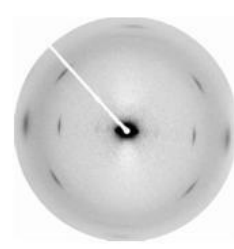

(e)
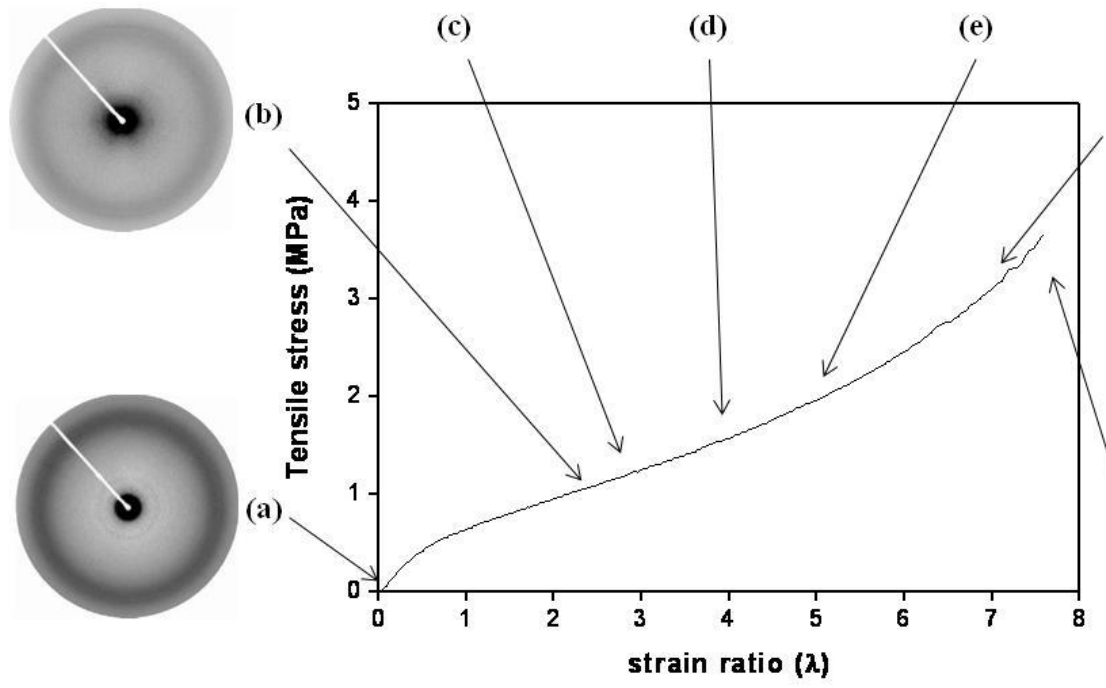

(f)

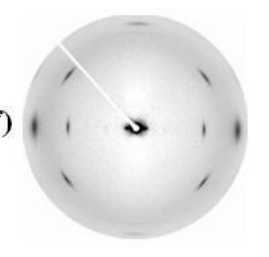

(g)

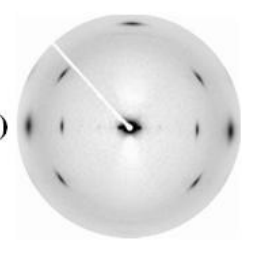


Figure 2. Tensile stress-strain curve of the vulcanized NR sample stretched up to $\lambda=$ 7.5. Insets correspond to WAXS patterns of NR upon stretching at room temperature at $\lambda=$ a) 0 , b) 2.5 , c) 3 , d) 4 , e) 5 , f) 7 and g) 7.5 .
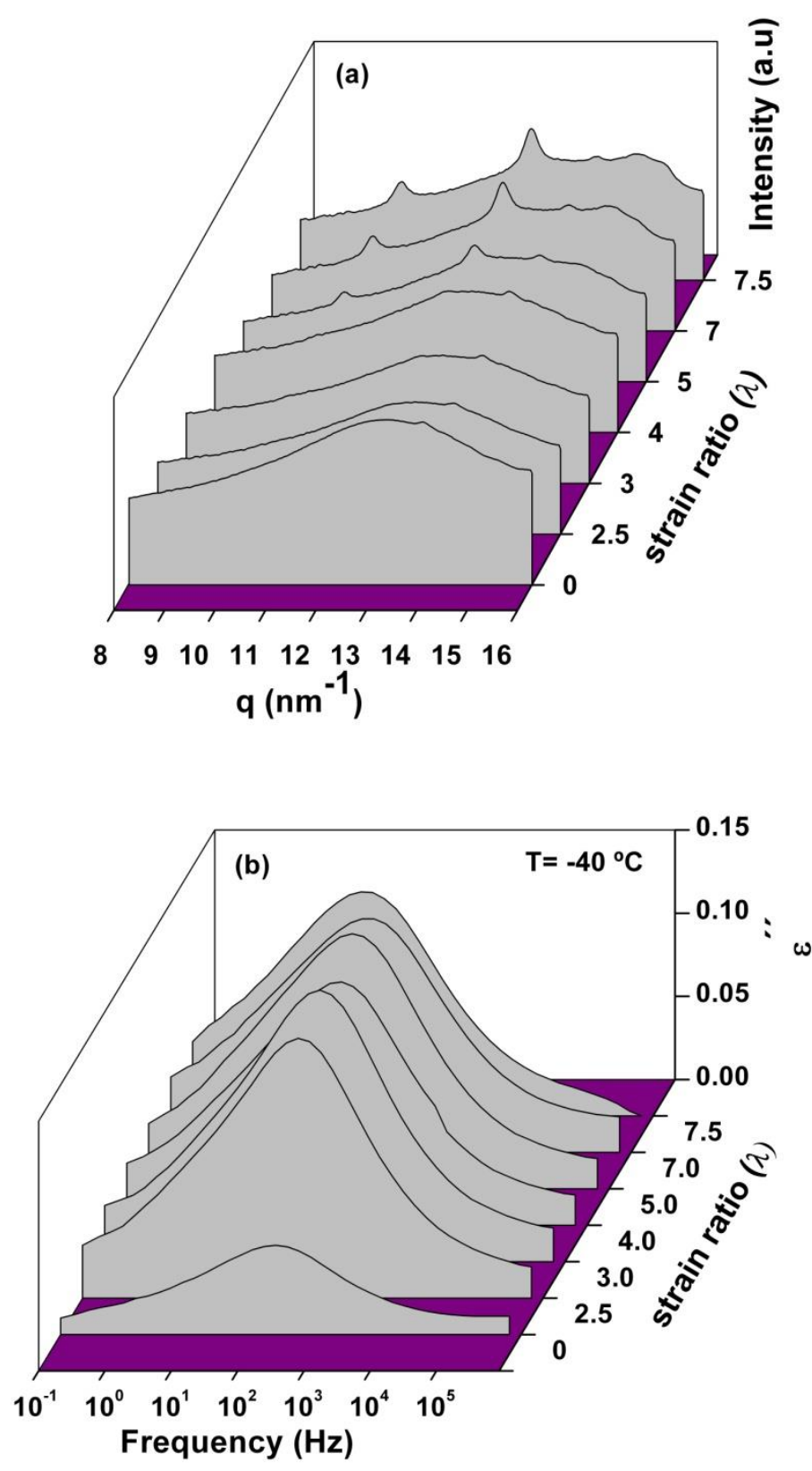

Figure 3. (a) Azimuthally integrated intensity of the WAXS patterns as a function of the scattering vector $\mathrm{q}$, and (b) Dielectric loss $\varepsilon^{\prime \prime}$ as a function of frequency, for vulcanized NR at different strain ratios.

\subsection{Strain induced changes in the segmental dynamics}


NR exhibits above the glass transition temperature, $T_{\mathrm{g}}$, two relaxation processes related to the segmental and chain dynamics in order of increasing temperature. ${ }^{14,}{ }^{15}$ However, in vulcanized NR the process associated to chain dynamics, referred to as normal mode ${ }^{16}$ is suppressed as a consequence of the crosslinking. Therefore vulcanized NR only exhibits above $T_{\mathrm{g}}=-64{ }^{\circ} \mathrm{C}$ one relaxation process, $\alpha$-relaxation, which manifests itself by a maximum in $\varepsilon^{\prime \prime}(F)$ (Figure 4). The segmental mode is assigned to the segmental motions of the polymer chains. ${ }^{14}$ In order to estimate the effect of stretching on the segmental dynamics of vulcanized NR with different strain ratios, the dielectric loss of every specimen was measured at $-40{ }^{\circ} \mathrm{C}$. At this temperature the $\alpha$-relaxation is well centered within the frequency window. The influence of uniaxial extension on the segmental dynamics of vulcanized NR can be visualized in Figure 3(b). Here, we have represented the dielectric loss spectra for vulcanized NR at the different strain ratios.

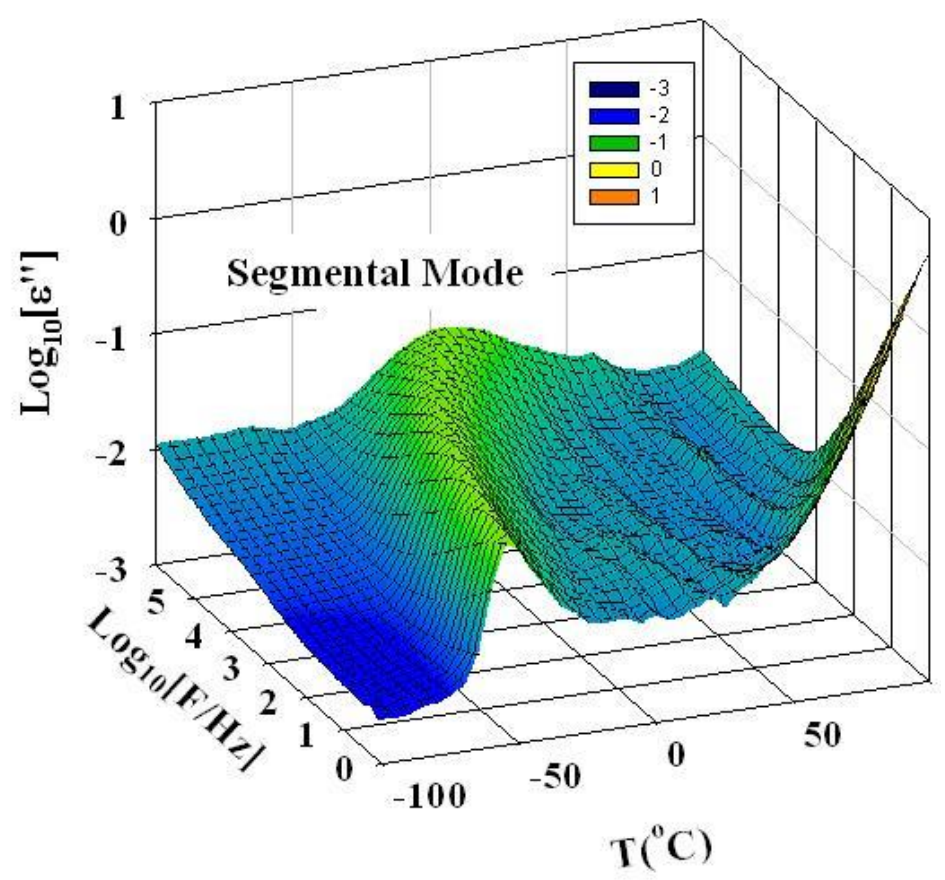

Figure 4. 3D representation of the frequency and temperature dependence of the dielectric $\operatorname{loss} \varepsilon^{\prime \prime}$ for the investigated vulcanized NR sample. ${ }^{14}$

\section{Discussion}

\subsection{Crystalline development upon strain}

Strain induced crystallization of vulcanized NR is a phenomena which has been widely reported by several authors elsewhere. ${ }^{2,8,11,25-27}$ In our case, the diffraction patterns observed upon strain correspond to that of semicrystalline vulcanized NR and are consistent with the monoclinic unit cell of polyisoprene. ${ }^{12,26}$ Specifically, the patterns present in the equator the reflections corresponding to the 200 and 120 planes. Different procedures have been proposed for the quantitative calculation of the strain- 
induced crystallinity from WAXS patterns. In this work, we based our analysis on a recent proposal published elsewhere ${ }^{26}$ which allows one to estimate a crystallinity index based on the equator diffraction peaks. ${ }^{20}$ In our case, the diffraction intensity near the equator was normalized and azimuthally integrated in a cake from $75^{\circ}$ to $105^{\circ}$ (see inset of Figure 5). As an example, Figure 5 represents the cake integrated intensity as a function of the scattering vector $\mathrm{q}$ for $\lambda=7.5$. The resultant profiles were deconvoluted considering the diffraction peaks of the 200 and 120 planes and the amorphous halo using the peakFIT ${ }^{\odot}$ software. The mass fraction crystallinity index, $X_{\mathrm{c}}$ was estimated according to:

$$
X_{C}=\frac{A_{C}}{A_{C}+A_{a}} * 100 \%
$$

Where $A_{\mathrm{c}}$ is the area below the 200 and 120 crystalline peaks and $A_{\mathrm{a}}$ is the area below the amorphous halo.
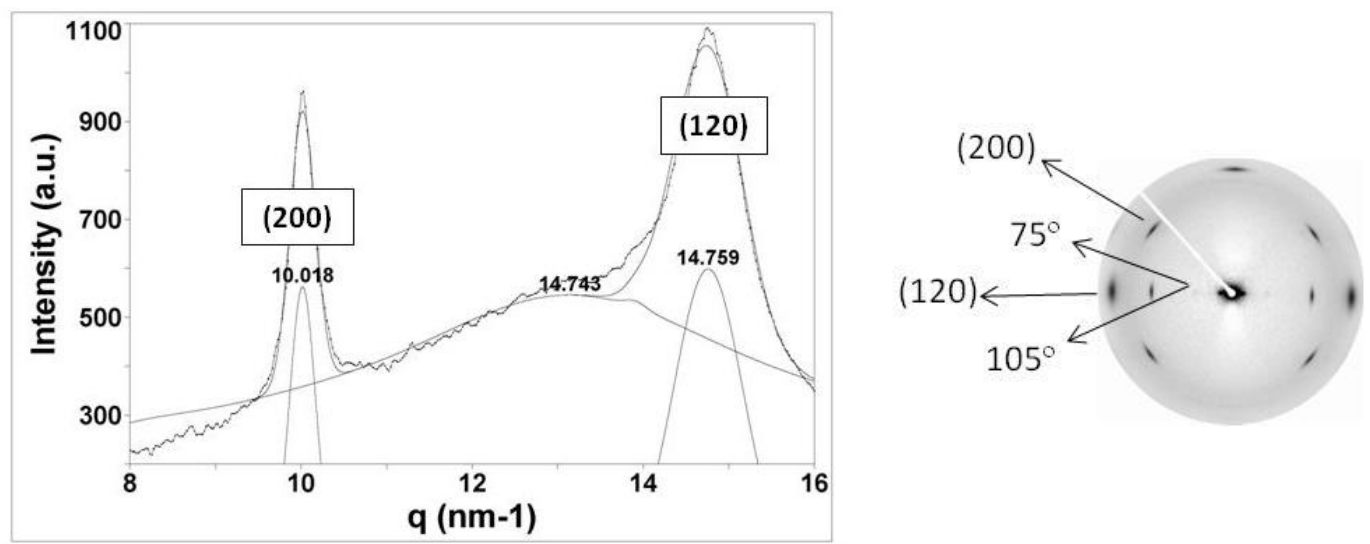

Figure 5. Equatorial cake integrated intensity as a function of the scattering vector $q$ taken from the WAXS pattern of stretched vulcanized NR at $\lambda=7.5$. Inset shows integration limits from $75^{\circ}$ to $105^{\circ}$.

Figure 6a) shows the calculated crystallinity index, $X_{\mathrm{c}}$ of all specimens as a function of the strain ratio. From these quantitative values, we can see that below a strain ratio of 2.5 no significant crystallinity is present. Strain-induced crystallization occurs above $\lambda=2.5$ and the crystallinity index increases gradually to reach a maximum value for a strain ratio of 7.5. These results are in accordance with the significant upturn of the strength in the stress-strain measurements (Figure 2). The polymer chains, which are initially coiled $\left(\mathrm{M}_{\mathrm{w}} \approx 10^{6} \mathrm{~g} / \mathrm{mol}\right)$, are stretched along the drawing direction and partially crystallize once a critical $\lambda=3$ is reached. Some of the tie molecule chains which have overflown from fibrillar crystallites between fibrillar and amorphous phases become part of the crystalline regions by the drawing process and hence, the degree of 
crystallinity increases by subsequent drawing. The effect of such amorphous to semicrystalline transition on the segmental dynamics of the stretched vulcanized NR samples will be discussed in the next paragraph.

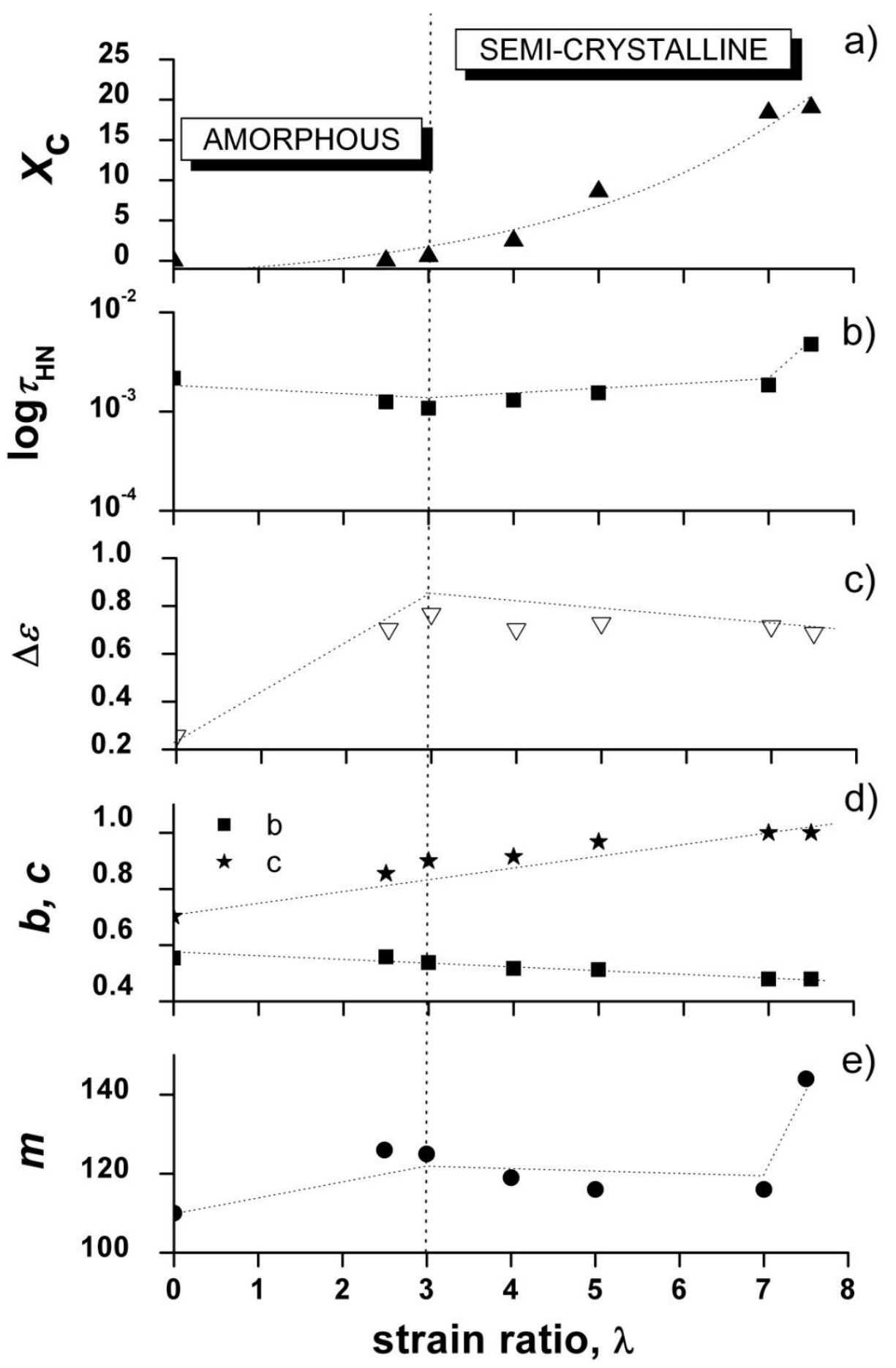

Figure 6. From top to bottom: a) Crystallinity index $X_{\mathfrak{c}}$, b) segmental relaxation time $\left.\tau_{\mathrm{HN}}, \mathrm{c}\right)$ dielectric strength $\left.\Delta \varepsilon, \mathrm{d}\right)$ dielectric shape parameters $(b, c)$, and e) fragility index $m$, as a function of strain ratio for the vulcanized NR samples. Dotted lines are guides for the eye. 


\subsection{Segmental dynamics dependence with strain}

The frequency dependence of $\varepsilon^{\prime \prime}$ for stretched vulcanized NR is displayed in Figure 7 at different strain ratios. Dielectric loss data for different stretch ratios can be fitted according to $\mathrm{HN}$ equation (eq. 1). The fit parameters $\left(\Delta \varepsilon, b, c\right.$ and $\left.\tau_{\mathrm{HN}}\right)$ obtained at $\mathrm{T}=-$ $40^{\circ} \mathrm{C}$ can be visualized in Figure 6. From the fittings, the most significant effect is a systematic deviation from the fitting of the experimental results at low frequencies (Figure 7). This effect has been reported by other authors and attributed to the Stearic acid present in the formulation of vulcanized NR. ${ }^{28}$ However, systematic dielectric studies done by us in samples with different formulation indicate that Zinc oxide has influence on this excess of dielectric loss. ${ }^{29}$

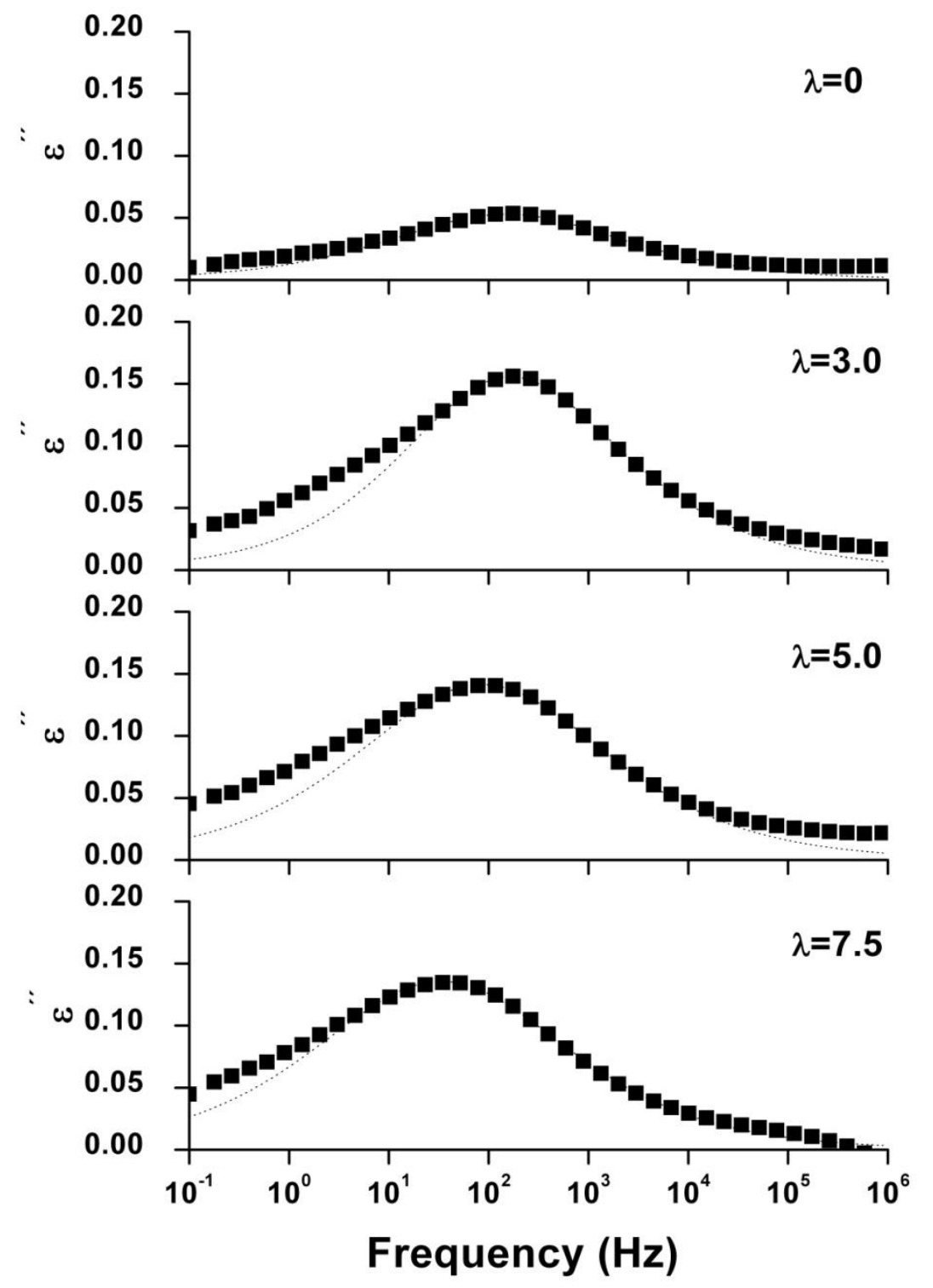


Figure 7. Frequency dependence of dielectric loss $\varepsilon^{\prime \prime}$ of NR samples stretched at different strain ratios as indicated on the plot in the region of the segmental mode ( $\mathrm{T}=-$ $\left.40^{\circ} \mathrm{C}\right)$. Dotted lines correspond to best $\mathrm{HN}$ fitting.

The dependence of the dielectric derived magnitudes can be separated into two regimes. In a first regime, for $\lambda<3$, no crystallization is evidenced. However a dramatic increase of the dielectric strength, $\Delta \varepsilon$, is observed. In the second regime, for $\lambda>3$, crystallization sets in and the variation of the shape parameters ( $b$ decreases and $c$ tends to the unit) with respect to the strain indicates a broadening of the relaxation for the segmental process. In this second regime, a decrease of $\Delta \varepsilon$ is observed. This behaviour is typical for the crystallization of semi-crystalline polymers where the crystalline domains slow down the segmental dynamics. ${ }^{17,18}$ Indeed, in our case the relaxation time, $\tau_{\mathrm{HN}}$, tends to increase slightly with the strain once crystallization appears. This effect can be expected since a reduced segmental mobility of the amorphous regions is imposed by the presence of the crystals.

In a first approach changes in $\Delta \varepsilon$ can be discussed based on the Frölich- Kirkwood (FK) equation ${ }^{30}$ which describes the temperature dependence of $\Delta \varepsilon$ as:

$\Delta \varepsilon \propto \frac{4 \pi \rho N_{A}}{9 k T M} g \mu^{2} \propto \mu_{e f f}^{2}$

Where $\rho$ is the density, $\mu$ is the dipole moment, $M$ is the molecular weight of the repeating unit, $N_{A}$ is Avogadro's number, $k$ is the Boltzmann constant and $g$ is the correlation factor which contains contributions of both inter and intra chain dipolar correlations and indicates the angular correlation between the dipole groups. The $g$ factor is frequently referred to as a reduction factor since the term $g \mu^{2}$ corresponds to the effective dipole moment, $\mu_{e f f}^{\boldsymbol{z}}$ " of the material. When no correlation among dipoles is expected, as in the gas state, $g$ approaches to 1 . In the first regime, $\lambda<3$, it is expected an increase of the density by stretching. However, the strong increase in $\Delta \varepsilon$ of about three times can not be solely explained by this fact. In vulcanized NR the dipole moments contributing to the segmental relaxation are mainly perpendicular to the polymer chain. ${ }^{16}$ In the non deformed state of vulcanized NR, as for most polymers, the variety of conformations partially cancel out some dipolar contribution. However, stretching can counteract this effect through ordering, causing the observed increase of the dielectric strength. The most favourable conformation of cis-1,4-polyisoprene is that with alternating $\mathrm{CH}_{3}$ - pendant groups in which dipoles should balance along the chain. ${ }^{31}$ However, recent molecular modelling ${ }^{32}$ has shown that the molecular asymmetric unit of ordered cis-1,4-polyisoprene is a di-isoprene in which the two isoprene residues have distinctive, not symmetrically related conformations. This suggests a possible increase of $\mu_{\text {eff }}$ upon stretching supporting the increment in $\Delta \varepsilon$ observed. In the second regime, $\lambda>3$, crystallization induces a decrease of the dielectric strength since dipoles included in the crystalline phase do not contribute to the segmental relaxation. Consequently the 
effect caused by stretching and that of crystallization producing opposite effects in $\Delta \varepsilon$ induce almost no change with stretching.

Figure 8 illustrates the relaxation time for the $\alpha$-relaxation, $\tau_{\max }$, as a function of the reciprocal temperature for different strain ratios. The $\alpha$ process exhibits the characteristic curvature of a Vogel-Fulcher-Tamman (VFT) dependence of the type:

$$
\tau_{\max }=\tau_{0} \exp \left(\frac{B}{T-T_{0}}\right) \propto\left(\frac{D T_{0}}{T-T_{0}}\right)
$$

Where

$$
\tau_{\max }=\frac{1}{2 \pi F_{\max }}=\tau_{\mathrm{HN}}\left[\sin \frac{b \pi}{2+2 c}\right]^{-1 / b}\left[\sin \frac{b c \pi}{2+2 c}\right]^{1 / b}
$$

$\tau_{0}$ and $B$ are empirical parameters, $T_{0}$ is the so-called ideal glass transition or Vogel temperature, which is generally $30-70 \mathrm{~K}$ below $T_{g}{ }^{33}$ and $D$ is the fragility strength. To reduce the effect of data fitting to the VFT equation over a limited frequency range, a value of $\log \tau_{0} \approx 14 \mathrm{~s}$ was assumed according to previous work. ${ }^{34}$ This assumption does not affect the quality of the data fit into the VFT equation, but reduces the dispersion among fitting parameters.

Several schemes have been proposed to classify the degree of relaxation time departure from the Arrhenius behaviour at the $\alpha$-relaxation process. ${ }^{35-37}$ The strong-fragile scheme proposed by Angell ${ }^{35}$ allows us to classify glass-forming systems on the basis of the temperature dependence exhibited by the relaxation time. Fragility ${ }^{38}$ is related to the manner in which temperature affects in general the properties of liquids and in particular their viscosity and molecular mobility above $T_{\mathrm{g}}$. Fragility can be quantified, ${ }^{37-}$ ${ }^{41}$ for example, by reference to the temperature dependence of the dielectric segmental relaxation time, $\tau$ as shown in eq. 4 . Also, a convenient method to estimate the fragility consists of calculating the fragility index $m$, defined as the apparent activation energy of either shear viscosity or relaxation time of the $\alpha$-relaxation ${ }^{37,41}$

$$
m=\frac{\partial \log (T)}{\partial\left(\frac{T_{g}}{T}\right)_{T=T_{g}}}=\frac{D T_{0} T_{g}}{\left(T_{g}-T_{0}\right)^{2} \ln 10}=16+\frac{590}{D}
$$

From the parameters presented in Table 2 we can see that the $T_{0}$ values for the stretched samples are higher than that of the undeformed vulcanized NR. Again two regimes can 
be recognized. Before crystallization, $\lambda<3$, there is a significant increase of about $10{ }^{\circ} \mathrm{C}$ in $T_{0}$. This effect contrasts with previous results reported for synthetic polyisoprene cross-linked with dicumyl peroxide. ${ }^{13}$ In this case no variation of the dielectric spectrum was observed upon stretching. However it is worth mentioning that in this study the cross-link density was $6.1 \times 10^{-5} \mathrm{~mol} / \mathrm{cm}^{3}$ while in our case, for vulcanized NR, is of $3.6 \times 10^{-3} \mathrm{~mol} / \mathrm{cm}^{3}$. This higher cross-link density of vulcanized NR can explain the different behaviour on both cases. For stretched vulcanized NR with $\lambda<3$ there is expected a significant reduction of the free volume, as compared with the undeformed sample, causing a significant increase of the glass transition temperature and consequently an increment of $T_{0}$. This effect is consistent with the observed increment on the fragility parameter, $m$, since as free volume reduces by stretching more cooperativity is needed in order to accomplish segmental motions.

For $\lambda>3$, as crystals develop, almost no variation in $T_{0}$ or in $m$ is observed. In strained vulcanized NR, it is proposed that crystallization starts by nucleation of short stretched chains which act as nuclei. The shorter chains among cross-links become fully stretched while the distribution of cross-links is not uniform keeping many network chains in the random-coil-like state. ${ }^{12}$ It is conceivable that during strain induced crystallization the shorter chains are progressively incorporated into the crystals while a significant amount of longer chains remains rather coiled. In this case, during this regime, the segmental dynamics of amorphous phase of vulcanized NR would not experience significant changes. Only for high strains, $\lambda=7.5$ in our case, the crystallinity is high enough as to provoke a new increment of the fragility index and of $T_{0}$.

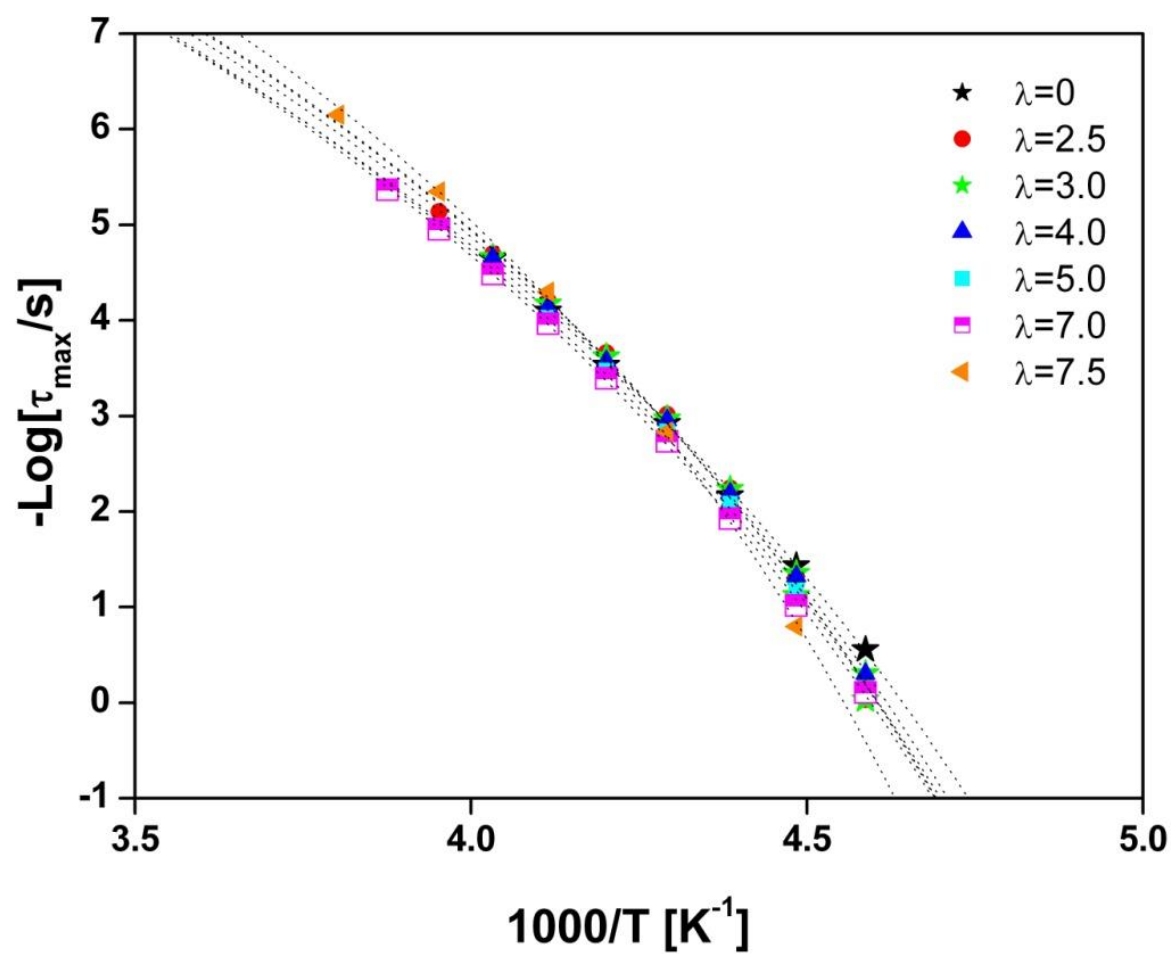


Figure 8. Activation plot of the average relaxation time for the segmental mode of NR stretched samples, as a function of strain ratio. The dotted lines represent the fits to eq. 4.

Table 2. VFT parameters for the segmental mode process of NR stretched at different strain ratios.

\begin{tabular}{ccc}
\hline Strain ratio $(\boldsymbol{\lambda})$ & $\boldsymbol{T}_{\mathbf{0}}, \mathbf{K}$ & $\boldsymbol{D}$ \\
\hline 0 & 148.7 & 6.3 \\
2.5 & 157.2 & 5.3 \\
3.0 & 156.8 & 5.4 \\
4.0 & 153.8 & 5.7 \\
5.0 & 152.7 & 5.9 \\
7.0 & 153.2 & 5.9 \\
7.5 & 165.3 & 4.6 \\
\hline
\end{tabular}

\section{5 . Conclusions}

The effects of strain-induced crystallization on the molecular dynamics of vulcanized NR have been studied by means of broadband dielectric spectroscopy and X-ray scattering techniques. The segmental dynamics of vulcanized NR is clearly affected by uniaxial stretching. Upon stretching three regimes are detected. For low strains, $\lambda<3$, stretching takes place without crystallization. However a dramatic increase of the dielectric strength, $\Delta \varepsilon$, is observed which can not be solely explained by an increase in density. We suggest that an increase of the effective dipole moment upon stretching is responsible for this increment in $\Delta \varepsilon$. Also, the expected reduction of the free volume by stretching causes an increase of the glass transition temperature and consequently an increment of $T_{0}$. This effect is accompanied by an increment on the fragility parameter, $m$, since as free volume reduces by stretching more cooperativity is needed in order to accomplish segmental motions. In the second regime, $3<\lambda<7.5$, crystals develop and the inclusion of segments into the crystalline phase counteracts the increment in $\Delta \varepsilon$ provoked by stretching. In agreement with previous models, our results indicate that during strain induced crystallization shorter chains are progressively incorporated into the crystals while a significant amount of longer chains remains rather coiled. In this case the segmental dynamics of the amorphous phase of vulcanized NR does not experience significant changes. Finally at high strains, $\lambda=7.5$, the crystallinity is high enough as to provoke a new increment of the fragility index and of $T_{0}$. 
Acknowledgement. This work was partially supported by MICINN grant MAT200803232 and MAT2009-07789. M. Hernández gratefully acknowledges the Mision Ciencia fellowship from the Venezuelan Ministry of Science and Technology.

\section{References}

1. Zhang, H. P.; Niemczura, J.; Dennis, G.; Ravi-Chandar, K.; Marder, M. Phys. Rev. Lett. 2009, 102, (24), 4.

2. Tosaka, M.; Kawakami, D.; Senoo, K.; Kohjiya, S.; Ikeda, Y.; Toki, S.; Hsiao, B. S. Macromolecules 2006, 39, (15), 5100-5105.

3. Le Cam, J. B.; Toussaint, E. Macromolecules 2010, 43, (10), 4708-4714.

4. $\quad$ Trabelsi, S.; Albouy, P. A.; Rault, J. Macromolecules 2002, 35, (27), 1005410061.

5. Tosaka, M. Macromolecules 2009, 42, (16), 6166-6174.

6. Trabelsi, S.; Albouy, P. A.; Rault, J. Macromolecules 2003, 36, (24), 9093-9099.

7. Poompradub, S.; Tosaka, M.; Kohjiya, S.; Ikeda, Y.; Toki, S.; Sics, I.; Hsiao, B. S. J. Appl. Phys. 2005, 97, (10), 9.

8. $\quad$ Carretero-Gonzalez, J.; Verdejo, R.; Toki, S.; Hsiao, B. S.; Giannelis, E. P.; Lopez-Manchado, M. A. Macromolecules 2008, 41, (7), 2295-2298.

9. Gent, A. N.; Zhang, L. Q. Rubber Chem. Technol. 2002, 75, (5), 923-933.

10. Toki, S.; Sics, I.; Ran, S. F.; Liu, L. Z.; Hsiao, B. S.; Murakami, S.; Senoo, K.; Kohjiya, S. Macromolecules 2002, 35, (17), 6578-6584.

11. Ikeda, Y.; Yasuda, Y.; Hijikata, K.; Tosaka, M.; Kohjiya, S. Macromolecules 2008, 41, (15), 5876-5884.

12. Tosaka, M.; Murakami, S.; Poompradub, S.; Kohjiya, S.; Ikeda, Y.; Toki, S.; Sics, I.; Hsiao, B. S. Macromolecules 2004, 37, (9), 3299-3309.

13. Lee, H. K.; Fragiadakis, D.; Martin, D.; Milne, A.; Milne, J.; Runt, J. Macromolecules 2010, 43, (7), 3125-3127.

14. Hernandez, M.; Carretero-Gonzalez, J.; Verdejo, R.; Ezquerra, T. A.; LopezManchado, M. A. Macromolecules 2010, 43, (2), 643-651.

15. Carretero-Gonzalez, J.; Ezquerra, T. A.; Amnuaypornsri, S.; Toki, S.; Verdejo, R.; Sanz, A.; Sakdapipanich, J.; Hsiao, B. S.; Lopez-Manchado, M. A. Soft Matter 2010, 6, (15), 3636-3642.

16. Boese, D.; Kremer, F. Macromolecules 1990, 23, (3), 829-835.

17. Sanz, A.; Nogales, A.; Ezquerra, T. A.; Soccio, M.; Munari, A.; Lotti, N. Macromolecules 2010, 43, (2), 671-679.

18. Nogales, A.; Ezquerra, T. A.; Denchev, Z.; Sics, I.; Calleja, F. J. B.; Hsiao, B. S. J. Chem. Phys. 2001, 115, (8), 3804-3813.

19. C.M. Blow, C. H., Rubber Technology and Manufacture. 2nd ed.; Butterworth Scientific: London, 1982; p 608.

20. Rueda, D. R.; Garcia-Gutierrez, M. C.; Nogales, A.; Capitan, M. J.; Ezquerra, T. A.; Labrador, A.; Fraga, E.; Beltran, D.; Juanhuix, J.; Herranz, J. F.; Bordas, J. Review of Scientific Instruments 2006, 77, (3).

21. Havriliak.S; Negami, S. Polymer 1967, 8, (4), 161-210.

22. Kremer, F.; Schönhals, A., Broadband Dielectric Spectroscopy. Springer: 2003.

23. Ward, I. M.; Sweeney, J., An Introduction to the Mechanical Properties of Solid Polymers. 2nd ed.; John Wiley \& Sons Ltd: West Sussex, 2004; p 382. 
24. Brydson, J. A., Plastic Materials. 4th ed.; Butterworth Scientific: London, 1982; p 800.

25. Murakami, S.; Senoo, K.; Toki, S.; Kohjiya, S. Polymer 2002, 43, (7), $2117-$ 2120.

26. Toki, S.; Hsiao, B. S.; Amnuaypornsri, S.; Sakdapipanich, J. Polymer 2009, 50, (9), 2142-2148.

27. Weng, G. S.; Huang, G. S.; Qu, L. L.; Nie, Y. J.; Wu, J. R. J. Phys. Chem. B 2010, 114, (21), 7179-7188.

28. Ortiz-Serna, P.; Diaz-Calleja, R.; Sanchis, M. J.; Floudas, G.; Nunes, R. C.; Martins, A. F.; Visconte, L. L. Macromolecules 2010, 43, (11), 5094-5102.

29. Hernandez, M.; Verdejo, R.; Ezquerra, T. A.; Lopez-Manchado, M. A. In preparation 2011.

30. Hedvig, P., Dielectric Spectroscopy of Polymers. Adam Hilger Ltd: Bristol, 1977.

31. Benedetti, E.; Corradini, P.; Pedone, C. Eur. Polym. J. 1975, 11, (8), 585-587.

32. Rajkumar, G.; Squire, J. M.; Arnott, S. Macromolecules 2006, 39, (20), 70047014.

33. Runt, J. P.; Fitzgerald, J. J., Dielectric Spectroscopy of Polymeric Materials. Fundamentals and Applications. American Chemical Society: Washington, DC, 1997; p 461.

34. Kramarenko, V. Y.; Ezquerra, T. A.; Sics, I.; Balta-Calleja, F. J.; Privalko, V. P. J. Chem. Phys. 2000, 113, (1), 447-452.

35. Angell, C. A. J. Non-Cryst. Solids 1991, 131, 13-31.

36. Roland, C. M.; Ngai, K. L. Macromolecules 1992, 25, (21), 5765-5768.

37. Bohmer, R.; Ngai, K. L.; Angell, C. A.; Plazek, D. J. J. Chem. Phys. 1993, 99, (5), 4201-4209.

38. Plazek, D. J.; Ngai, K. L. Macromolecules 1991, 24, (5), 1222-1224.

39. Green, J. L.; Ito, K.; Xu, K.; Angell, C. A. J. Phys. Chem. B 1999, 103, (20), 3991-3996.

40. Richert, R.; Angell, C. A. J. Chem. Phys. 1998, 108, (21), 9016-9026.

41. Angell, C. A. Polymer 1997, 38, (26), 6261-6266. 